\title{
Development of predictive prognostic nomogram for NECs of rectum on population-based exploration
}

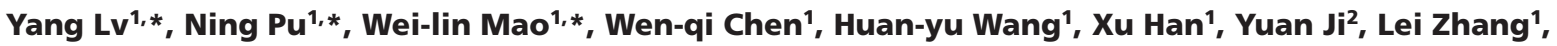 \\ Da-yong Jin', Wen-Hui Lou' ${ }^{1}$ and Xue-feng $X_{\mathbf{u}^{1}}$ \\ ${ }^{1}$ Department of General Surgery, Zhongshan Hospital, Fudan University, Shanghai, China \\ ${ }^{2}$ Department of Pathology, Zhongshan Hospital, Fudan University, Shanghai, China \\ Correspondence should be addressed to X Xu or W Lou: xuefengxu87@aliyun.com or lou.wenhui@zs-hospital.sh.cn \\ *(Y Lv, N Pu and W Mao contributed equally to this work)
}

\begin{abstract}
Aim: We aim to investigate the clinical characteristics of the rectal NECs and the prognosis-related factors and construct a nomogram for prognosis prediction. Methods: The data of 41 patients and 1028 patients with rectal NEC were retrieved respectively from our institution and SEER database. OS or PFS was defined as the major study outcome. Variables were compared by chi-square test and $t$-test when appropriate. Kaplan-Meier analysis with log-rank test was used for survival analysis and the Cox regression analysis was applied. The nomogram integrating risk factors for predicting OS was constructed by $\mathrm{R}$ to achieve superior discriminatory ability. Predictive utility of the nomogram was determined by concordance index (C-index) and calibration curve. Results: In the univariate and multivariate analyses, tumor differentiation, N stage, M stage and resection of primary site were identified as independent prognostic indicators. The linear regression relationship was found between the value of $\mathrm{Ki}-67$ index and the duration of OS $(P<0.05)$. Furthermore, the independent prognostic factors were added to formulate prognostic nomogram. The constructed nomogram showed good performance according to the C-index.

Conclusions: Contrary to WHO classification guideline, we found that the rectal NEC diseases are heterogeneous and should be divided as different categories according to the pathological differentiation. Besides, the nomogram formulated in this study showed excellent discriminative capability to predict OS for those patients. More advanced predictive model for this disease is required to assist risk stratification via the formulated nomogram.
\end{abstract}

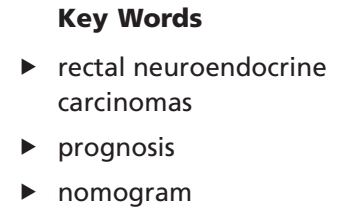

Endocrine Connection (2018) 7, 1178-1185

\section{Introduction}

Rectal neuroendocrine neoplasms (rectal NENs) are a series of heterogeneous neoplasms, which make up the third commonest neoplasms of rectum $(1,2)$. The incidence and prevalence of rectal NENs has been increasing dramatically over the last decades (1). The 2010 WHO classification utilized tumor proliferative ability (including Ki-67 proliferation index and mitotic rate) to divide NENs into three pathological grades: low grade (G1), intermediate grade (G2) and high grade (G3). According to the 2010 WHO classification, high-grade G3 rectal neuroendocrine carcinomas (NECs) were defined as a tumor with Ki-67 proliferation index greater than $20 \%$

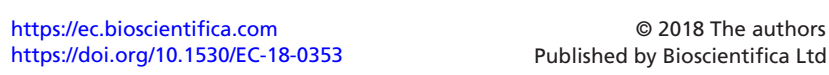


or mitotic rate greater than 20 per 10 high power fields (3). NECs are clinically related to aggressive tumor behavior and devastating prognosis (4). However, study has (5) proved that NECs could also demonstrate heterogeneous oncological behavior with a relatively wide spectrum of differentiation degree. Increasing evidence indicate that the current WHO G3 category may not sufficiently explain the difference among the highly, well-moderately and poorly differentiated NECs due to the lack of welldefined histologic criteria as well as the small volume of the case publications (6).

Recently, a population-based multiple-centered study reported that rectal NECs accounted for only 3.56\% of the gastroenteropancreatic NENs (GEP-NENs) in China (7). Given the rarity and the indolent process of the high-grade rectal NECs, the research of NEC should better be studied in large, population-based registries with considerable long-term follow-up (8). The Surveillance, Epidemiology, and End Results (SEER) program of the National Cancer Institute provides authoritative information on cancer statistics, which have been widely used to explore the clinical relationship and survival outcomes in patients with cancer (9). The data from SEER program showed that incidence of rectal NECs has increased from 0.2/100,000 to $1.2 / 100,000$ during the past three decades (1). However, effective analysis of critical issues for rectal NECs were lacking in a population-based scenario, as well as the need of the specific clinical significance of prognosis-related factors (10). Considering the heterogeneous morphology and biology, it is important to recognize clinical characteristics and outcomes of G3 rectal NECs; thus, in this paper, we aim to analyze the clinical characteristics of the rectal NECs and the prognosis-related factors.

Nomogram is a reliable tool to quantify risk of tumor by illustrating important factors for oncological prognosis. However, clinical application of nomogram in rectal NEC patients is still not available. In this study, we use data derived from the SEER database as the primary cohort to identify related prognostic factors affecting overall survival (OS) of rectal NEC patients and to construct nomogram for prognosis prediction for these patients. Besides, the internal and external validations were also performed to determine the specific prognostic capability.

\section{Methods and materials}

\section{Patients and data collection}

The study was approved by Ethical Committee of Fudan University, and it followed the ethical guidelines of the
Declaration of Helsinki. Consent has been obtained from each patient after full explanation of the purpose and nature of all procedures. Clinical characteristics of 41 consecutive patients with pathologically diagnosed WHO G3 rectal NECs from January 2000 to July 2014 were retrieved from Zhongshan Hospital of Fudan University in China. Patients who had previous or concomitant history of other malignant tumor or documented hereditary syndromes like multiple endocrine neoplasia type 1 (MEN-1) were excluded. Institutional review board approval was obtained in this study.

We collected data of patients with rectal NECs from the SEER database. We selected all NENs of the rectum (site code: C20.9) from the SEER database. The following ICD-O-3 histology code applied to identify neuroendocrine carcinomas was 8246-2 and 8246-3. Patients diagnosed with positive pathology from 1998 to 2015 were included in this study. Patients with a history of unclear clinical information were excluded, as is shown in flowchart (Fig. 1).

Data containing age at diagnosis, sex, date of initial diagnosis, tumor differentiation, tumor size, nodal status, location of distant metastasis and duration of progressive survival (PFS) and OS were retrieved separately from our institution and SEER database. Surgery types including abdominal peritonium resection (APR), resection of the

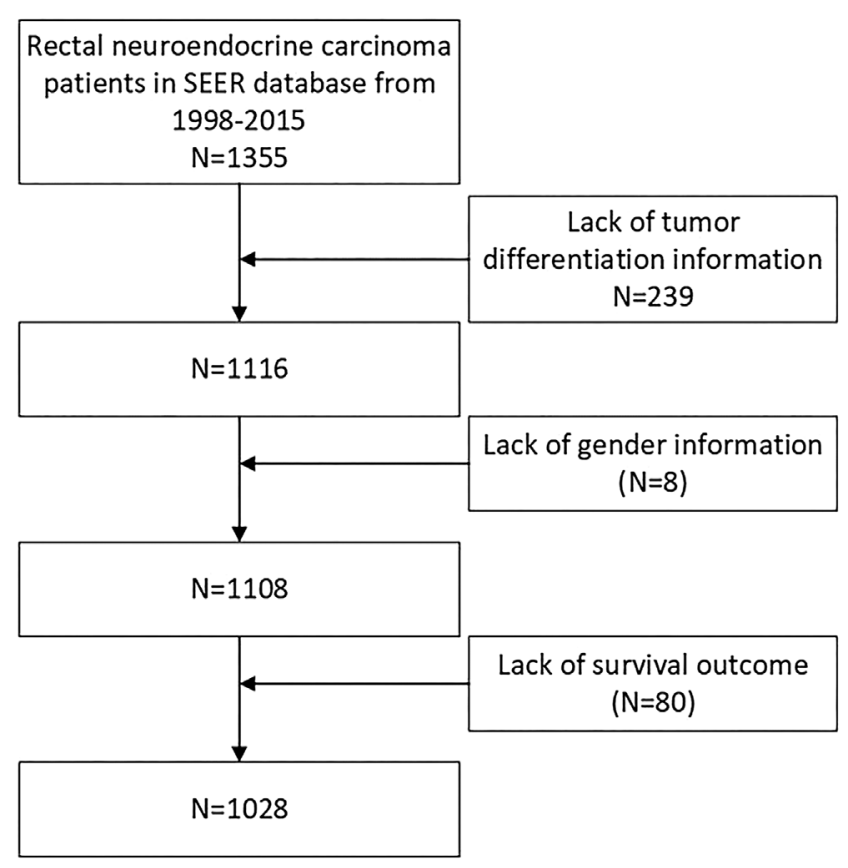

Figure 1

Flowchart of included population in this study. Rectal NECs, rectal neuroendocrine carcinomas; SEER, Surveillance, Epidemiology, and End Results. 
rectal cancer through abdomen (Dixon) and resection of the rectal cancer with distal closure and proximal stoma (Hartmann) procedures were also collected. Furthermore, other information including clinical manifestations, specific Ki-67 proliferation index and mitotic rate were collected in only Chinese patients, because the Ki-67 index and mitotic rate were not available in SEER database. In our center, all the cases and pathological sections were blindly re-evaluated by an independent pathologist, according to the site of origin, criteria of the WHO, graded according to the ENETS proposal for grading and staging of NENs (11).

\section{Follow-up}

The patients in our center receive a periodical follow-up via the outpatient clinics or phone call. The information of the tumor progression was also recorded accordingly. The follow-up period was as follows: the first follow-up was conducted within 2-3 months after operation and the subsequent follow-up cycle usually ranges from 3 to 6 months or even shorter if tumor relapse or metastasis was suspected. The data of OS and relapse/metastasis time were documented. The duration of OS was calculated from the date of operation to the last follow-up or cancerspecific death. The relapse time was computed from the date of operation to recurrence. In our study, the median follow-up duration was $52.17 \pm 32.72$ months (range from 3 to 121 months).

\section{Statistical analysis}

Statistical analyses were performed using the SPSS statistical package (19.0; SPSS Inc). Pearsons Chi-square test and Fishers exact test were used to compare proportions when appropriate, whereas means were compared using a nonparametric test: Mann-Whitney test or the KruskalWallis test was used when there were more than two means. Pearson correlation and nonlinear exponential regression analysis were also carried out. OS analyses were carried out using the Kaplan-Meier method and the results were compared using a log-rank test. A multivariable Cox proportional hazards model predicting OS was performed using backward stepwise selection. Risk factors were expressed as the hazard ratio (HR, 95\% confidence interval (CI)). Statistical significance was defined as $P$ value less than 0.05 .

The predictive nomogram was formulated on the basis of a multivariate analysis using the package of rms in $\mathrm{R}$ version 3.3.0 (http://www.r-project.org/). The validation and discrimination of the nomogram was determined by the Harrell concordance index (C-index) as an index of model performance. Higher C-index values indicate better discrimination: a value of 0.5 defines no predictive discrimination, whereas a value of 1.0 defines perfect discrimination of individuals with different outcomes. The exact $\mathrm{R}$ code formulating the nomogram and calibration curve is shown in the Supplementary data (see section on supplementary data given at the end of this article).

Table 1 Common clinical characteristics of rectal NECs patients in our center and in SEER database.

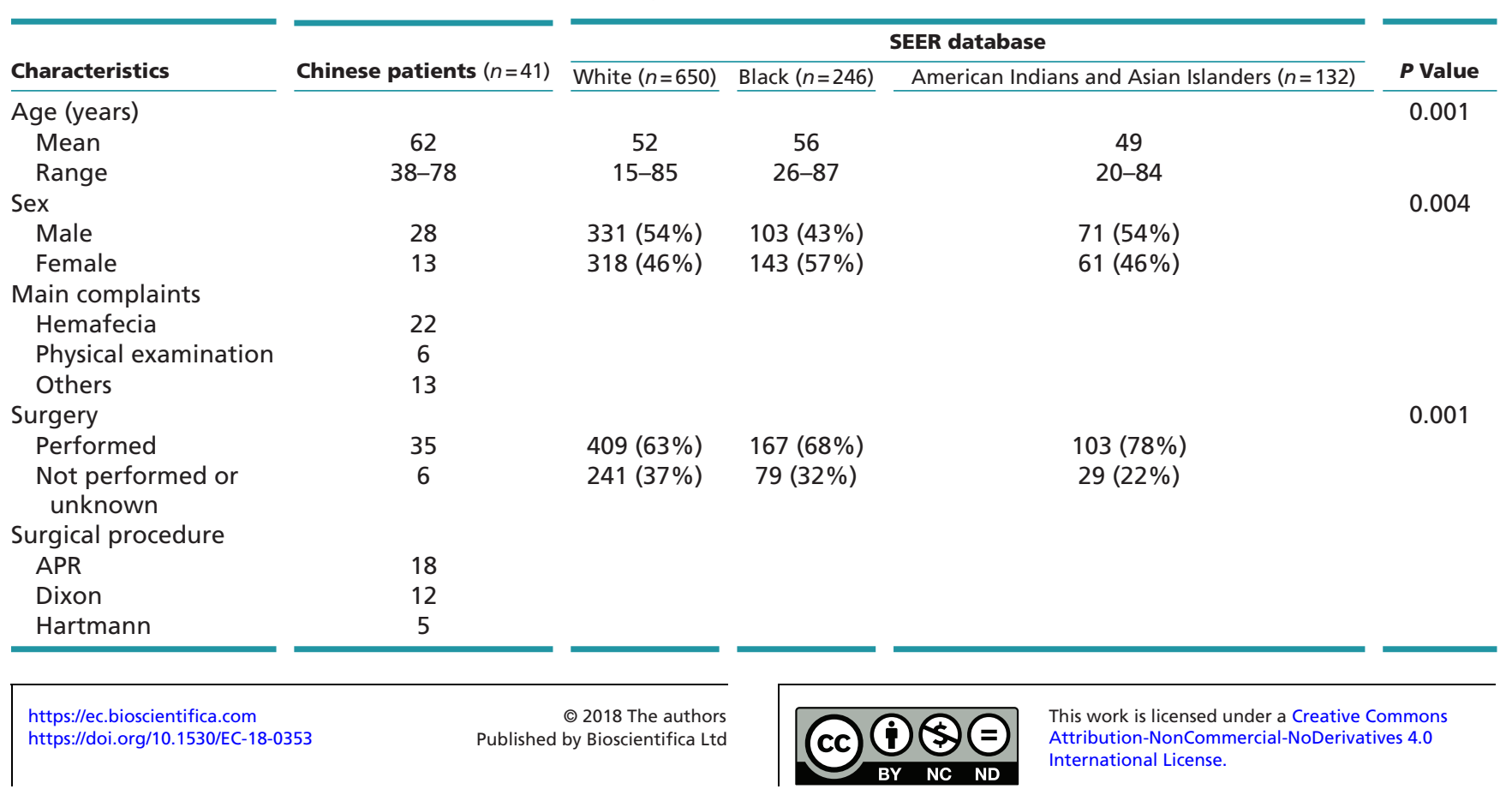




\section{Results}

\section{Comparison of the clinical characteristics of rectal NECs between SEER cohort and our center}

In this study, the clinical data of 41 patients and 1355 patients of rectal NECs disease were collected respectively from our center and SEER database, 1028 patients from SEER database who met the inclusion criteria were incorporated into this study (Fig. 1). Table 1 summarizes the common clinical characteristics of those patients. The median age was 63, 54, 56 and 49 years old in our center, Caucasian, black people and American Indians respectively. Among the SEER cohort, patients from California accounted for the majority (428 out of 1028 patients). Except the black patients, males were shown more statistically common in the prevalence of rectal NECs disease. Among all the patients, the rate of surgery performed on the primary lesion was $85,63,68$, and $78 \%$ in our patients, Caucasian, black and American Indians patients respectively. In our cohort, the APR procedure was performed in 18 patients (51\%), followed by Dixon in 12 patients (34\%) and Hartmann in five patients (14\%). The mean Ki-67 index was $51 \pm 22$ (range from 20 to 100 , coefficient of variation $(\mathrm{CV})$ is $42 \%)$. Compared with Chinese patients, stage I and stage IV from AJCC 7th edition were more commonly found in Caucasian, black and American Indian patients. In our patients, according to the pathological examination, $37 \%$ of the rectal NECs tumor was functional, and $63 \%$ of patients were non-functional; the most common presenting symptom was hemafecia (54\%). Regarding the tumor differentiation, well-differentiated degree was found in $24 \%$ of white patients, $13 \%$ of black patients and $8 \%$ of American Indian patients. Compared to this, 51\% of our patients were found in moderate differentiation.

Table 2 Characteristics of patients in SEER cohort: univariate and multivariate analysis.

\begin{tabular}{|c|c|c|c|c|c|}
\hline \multirow[b]{2}{*}{ Characteristics } & \multirow[b]{2}{*}{ Patients } & \multicolumn{4}{|c|}{ Overall Survival } \\
\hline & & Univariate analysis & Multivariate analysis & Multivariate HR & $95 \%$ Confidence interval $(\mathrm{Cl})$ \\
\hline Total & 1028 & & & & \\
\hline Gender & & 0.55 & NA & 0.89 & $0.664-1.203$ \\
\hline Males & 609 & & & & \\
\hline Females & 419 & & & & \\
\hline Age (years) & & 0.001 & 0.46 & & \\
\hline $15-39$ & 68 & & & & \\
\hline $40-59$ & 546 & & & & \\
\hline $60-79$ & 336 & & & & \\
\hline $80+$ & 78 & & & & \\
\hline Differentiation & & 0.001 & 0.000 & 1.28 & $1.16-1.42$ \\
\hline Well-differentiated & 563 & & & & \\
\hline Moderately-differentiated & 125 & & & & \\
\hline Poorly differentiated/undifferentiated & 340 & & & & \\
\hline T classification & & 0.001 & 0.053 & 1.19 & $1.06-1.33$ \\
\hline $\mathrm{T} 2$ & 44 & & & & \\
\hline T3 & 110 & & & & \\
\hline $\mathrm{T} 4$ & 60 & & & & \\
\hline $\mathrm{N}$ classification & & 0.001 & 0.018 & 0.68 & $0.38-1.22$ \\
\hline No & 216 & & & & \\
\hline N1 & 648 & & & & \\
\hline N2 & 164 & & & & \\
\hline M classification & & 0.000 & 0.003 & 0.38 & $0.22-0.67$ \\
\hline Mo & 832 & & & & \\
\hline M1 & 196 & & & & \\
\hline TNM stage & & 0.000 & 0.000 & 1.54 & $1.30-1.82$ \\
\hline I & 516 & & & & \\
\hline II & 88 & & & & \\
\hline III & 143 & & & & \\
\hline IV & 281 & & & & \\
\hline No & 412 & & & & \\
\hline $\begin{array}{l}\text { https://ec.bioscientifica.com } \\
\text { https://doi.org/10.1530/EC-18-0353 }\end{array}$ & $\begin{array}{r}\text { (c) } 2 C \\
\text { ublished by } B\end{array}$ & $\begin{array}{l}18 \text { The authors } \\
\text { ioscientifica Ltd }\end{array}$ & & $\begin{array}{l}\text { This work is licen } \\
\text { Attribution-NonC } \\
\text { International Lice }\end{array}$ & $\begin{array}{l}\text { ed under a Creative Commons } \\
\text { ommercial-NoDerivatives } 4.0 \\
\text { nse. }\end{array}$ \\
\hline
\end{tabular}




\section{Independent prognostic factors of rectal NECs in our center and in SEER database}

For rectal NECs, the median OS from SEER database was $32 \pm 36$ months, ranging from 1 to 337 months. All the 1028 patients were included in univariate and multivariate analysis to determine the prognosisrelated factors, which was demonstrated in Table 2 . The univariate analysis found that different levels of age $(P=0.001)$, differentiation $(P=0.001)$, TNM classification $(P=0.000)$ and the resection of primary lesion $(P=0.001)$ were statistically associated with different durations of OS. From the multivariable analysis of OS, grade $(P<0.001$, $\mathrm{HR}=1.28 ; 95 \%$ confidential interval (CI): 1.16-1.42), $\mathrm{N}$ stage $(P=0.018, \mathrm{HR}=0.68 ; 95 \%$ confidential interval (CI): $0.34-1.22), M$ stage $(P=0.000, \mathrm{HR}=1.54 ; 95 \% \mathrm{CI}$ : $1.30-1.82)$ and resection of tumor $(P=0.001, \mathrm{HR}=0.43$; 95\% CI: 0.30-0.62) were regarded as independent risk indicators for tumor OS.

In our data, the common characteristics were already displayed in Table 1 . The mean OS was $44 \pm 32$ months, ranging from 3 to 144 months (CV is $73 \%$ ); the mean PFS was $12.00 \pm 11.31$ months, ranging from 2 to 36 months (CV is $94 \%$ ). Table 3 summarizes the result of univariate and multivariate analyses. For our patients, the 5-year OS rate was separately 75 and $20 \%$ for the variant AJCC T1/T2 and T3/T4 classification, compared with 45 and 30\% for the AJCC N0/N1 and N2 classification groups (logrank $\chi^{2}=18.08, P<0.05$ and $\log$-rank=27.92, $P=0.00$, respectively). From the multivariate survival analysis, the N, M stage and grade were indicated as the independent factors.

As one of the two important factors for the WHO classification of NETs disease, the value of Ki-67 was also evaluated through this study. Of all the rectal NECs patients, the mean Ki-67 index was $51 \pm 22$, ranging from 20 to 100 . To determine the clinical relationship between the Ki-67 index and OS, progression free survival (PFS) in rectal NECs, the scatter diagram of OS, PFS and Ki-67 distribution were developed using regression analysis. Figure 2A illustrated that there was a nonlinear exponential regression between the value of Ki-67 index $(X)$ and $O S(Y)$ level $\left(Y=0.67{ }^{*} X+78.37, F=9.78, R=0.20\right.$, $P=0.0033)$. However, same statistical correlation did not exist for the duration of PFS $(Y=0.04 * X+15.06$, $F=0.20, \quad P=0.65$ ) (Fig. 2B). Furthermore, of different differentiation the mean value of Ki-67 index was $38 \pm 24$ of well differentiation ( $\mathrm{CV}$ is $61.91 \%$ ), $54 \pm 21$ of moderate differentiation (CV is $39 \%$ ) and $60 \pm 16$ of poor differentiation (CV is $27 \%)$, respectively $(P=0.63)$.

\section{The construction and validation of prognostic nomogram for os}

A prognostic nomogram that combined variant remarkable independent predictors for OS in patients with rectal NECs was developed in primary cohort which was shown in Fig. 3A. The developed nomogram was based on the significant risk factors obtained using

Table 3 Univariate and multivariate survival analysis of rectal neuroendocrine carcinomas survival based on different factors in our center. 2000-2014 ( $n=41)$.

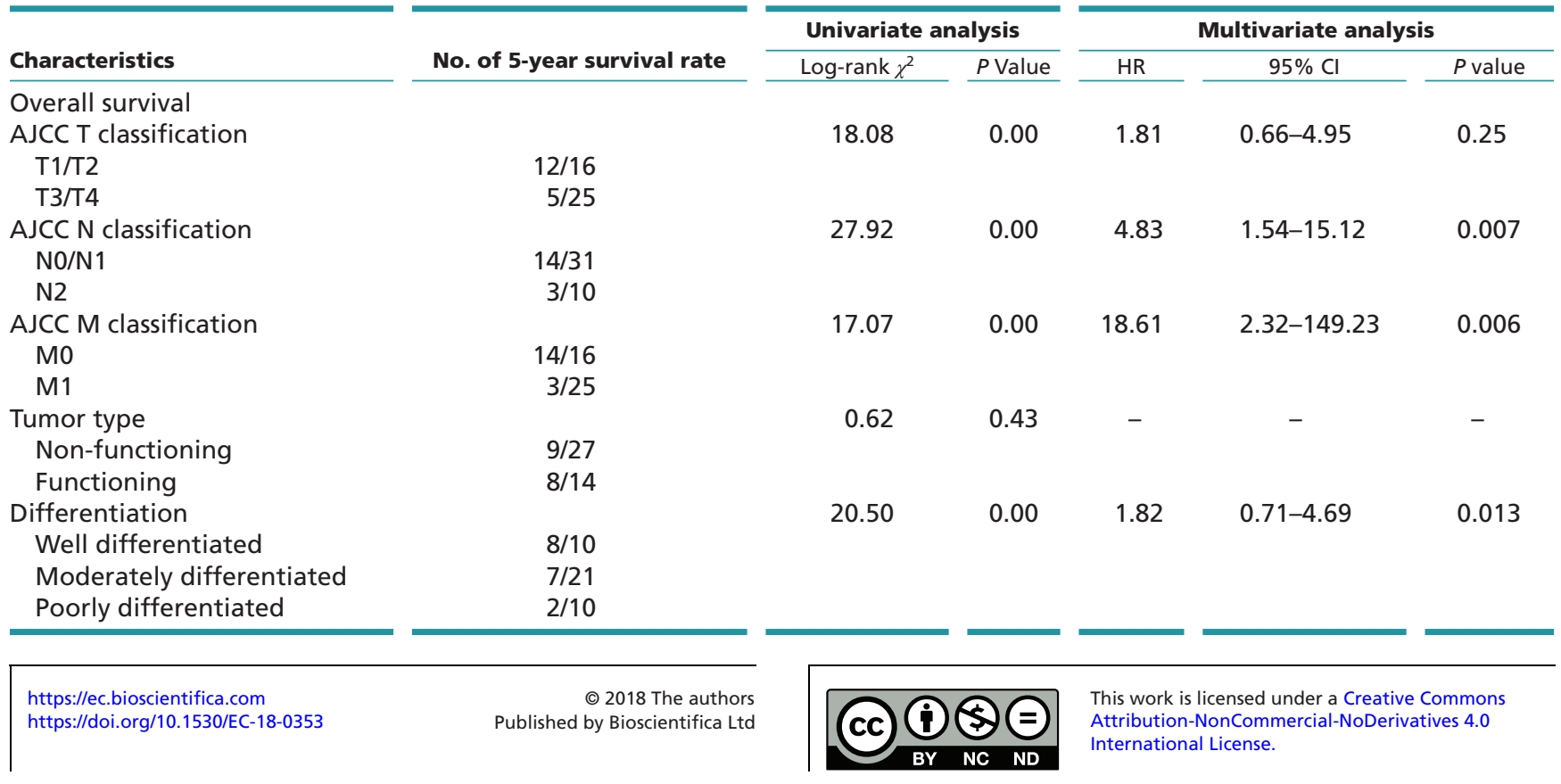



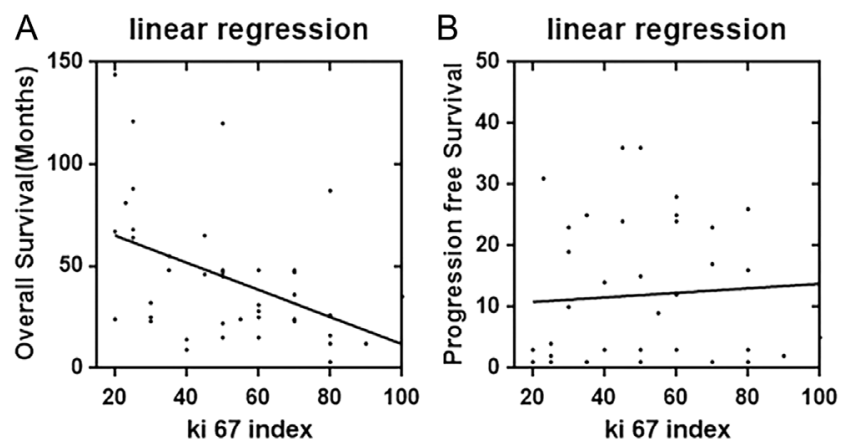

Figure 2

The correlation between survival and Ki-67 index levels in rectal NECs patients; $(\mathrm{A})$ the Ki-index value correlated with OS and there was a linear regression between them. (B) The Ki-index correlated with PFS with no statistical significance.

multivariate analyses. The 1-, 2- and 3-year probability of OS was estimated by summing the total points of all four independent predictors from the SEER database with the C-index of 0.91 . The internal and external calibration curve for the probability of OS rate showed optimal consistency and fit well between the nomogram-based prediction and actual observations, which was demonstrated in Fig. 3B, C, D, E and F.

\section{Discussion}

It is reported that the NETs of the rectum account for about $20 \%$ of the gastrointestinal NETs (12). However, majority of the rectal NETs are WHO G1 and G2, while the rectal NECs was rare and there were very few studies related to its clinical characteristics. Aytac (13) has reported that rectal NEC compromised about $0.33 \%$ of all colorectal malignancies. Fan (7) has reported from a nationwide study during 10 years that only 73 of 2049 GEP-NENs patients were rectal NEC patients in China.
Given the rarity of rectal NECs disease, the clinical management can usually be difficult to be optimal. Thus, the prognosis-related analysis of this cancer should be studied in large, population-based registries with relatively long-term follow-up. The National Cancer Institute's SEER database was a comprehensive source of population-based information which started from 1973. To determine the long-term clinical outcomes and prognosis-related factors of rectal NECs, we also collected a series of patients with rectal NECs from SEER database.

Through the univariate analysis and following multivariate analysis, we found that the differentiation, $\mathrm{N}$ stage, $\mathrm{M}$ stage, surgery are independent prognostic factors $(P<0.05)$, which were basically consistent with other kinds of tumors $(14,15)$. In particular, tumor differentiation and TNM stage were commonly regarded as important factors in many cancers and some recent reports even combined these stages with the levels of biomarkers to improve the prediction power. From our study, N, M stage and tumor differentiation have proven to influence the prognosis and were added into the construction of nomogram with a decent capability. Thus, we strongly recommend the inclusion of tumor differentiation in future rectal NEC staging systems.

Because NET is heterogeneous with respect to survival of individual patients, prediction just using the TNM staging system or WHO classification is imprecise (2). For those with rectal NEC disease, a prognostic prediction model seems to be rather valuable and critical and has not been reported previously (16). Furthermore, nomogram has been regarded as reliable tool to quantify risk by illustrating important factors for oncological prognosis (17). Despite several advances in the field of OS prediction for NECs patients (10), a nomogram has not been developed. In our study, we constructed a nomogram to predict the prognosis for rectal NEC patients. Presenting
A
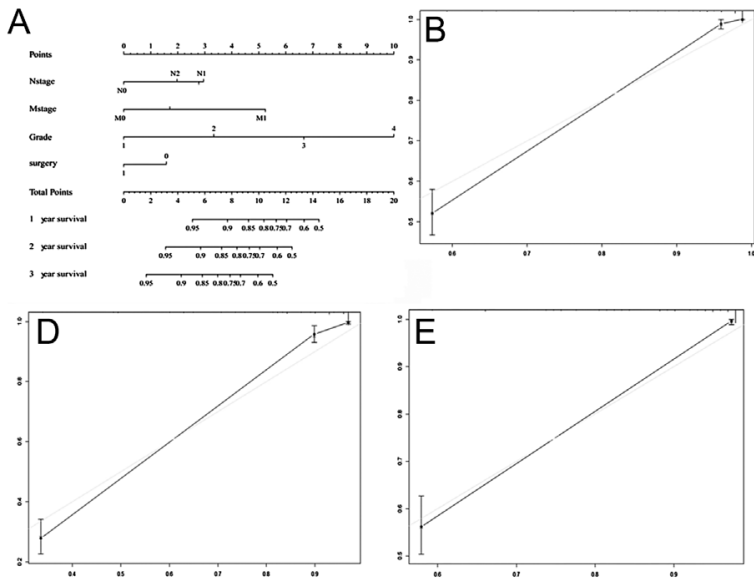
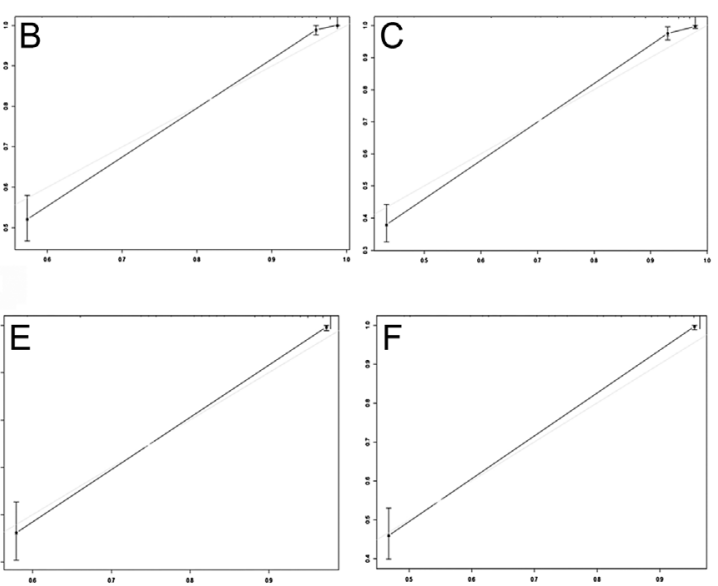

Figure 3

(A) Nomogram predict OS of patients with rectal NECs. For validating the nomogram, the sum of each predictor point was charted on the total points axis, and the estimated OS rate were performed through plotting a vertical line from the charted total point's axis straight down to the same OS rate axis. Besides, the prognostic nomogram constructed respectively using the data from the SEER database (A). The calibration curve for predicting overall survival of patients at 1 year $(B), 2$ year $(C)$ and 3 year (D) predicting OS in the SEER cohort, and predicting OS of patients at 2 year (E), 3 year (F) in the validation cohort. https://ec.bioscientifica.com https://doi.org/10.1530/EC-18-0353 (c) 2018 The authors Published by Bioscientifica Ltd
This work is licensed under a Creative Commons Attribution-NonCommercial-NoDerivatives 4.0 International License. 
the clinical data of more than 1000 cases, the internal and external calibration curves were constructed separately for the validation of the prediction model. OS rates were evaluated through the nomogram based on several significant factors (i.e., surgery, differentiation, $\mathrm{N}$ stage, $\mathrm{M}$ stage). The $\mathrm{C}$-index revealed the capability of nomogram to predict OS rates of patients with rectal NECs. The C-index of the formulated nomogram was 0.91 in SEER source, which was excellently predictive (18). In the validation cohort (data from our institution), our constructed nomogram was verified with better net benefit along with wider range of threshold probability and improved performance for predicting 1-, 2- and 3-year OS in terms of preferable C-index.

It is believed that tumor grade could reflect inherent tumor aggressiveness. The 2010 WHO classification divided the NETs into three pathological grades according to the value of Ki-67 proliferation index and mitotic rate: G1, G2 and G3. Of them, WHO G3 NEC was defined as a tumor with $\mathrm{Ki}-67$ proliferation index greater than $20 \%$ or mitotic rate greater than 20 per 10 high power fields (19). Thus, the Ki-67 index and mitotic rate were regarded as the prognosis-oriented factors. Besides, mounting evidence indicated that Ki-67 index functions more effectively than mitotic rate (19). However, due to the wide spectrum of Ki-67 index in G3 classification, the prognosis of the whole series of rectal NEC patients would not only be influenced by mere one or two markers. In the current study, the nonlinear exponential regression was found between the value of Ki-67 index and OS $(P=0.0033)$, which indicated that the Ki-67 index still played an important role on the prognosis prediction; in contrast, the duration of progression was not in statistical correlation with Ki-67 index. Furthermore, recently, there were emerging evidence suggesting that the current WHO G3 category may not sufficiently distinguish highly proliferative tumorous activity from well-moderate invasiveness due to the lack of well-defined histologic criteria (20). For all the G3 rectal NECs patients in our center, the differentiation was the independent prognostic factor for OS. To further investigate the correlation between Ki-67 and differentiation, our study found that the poor differentiation was trendy to express higher value of Ki-67 index $(P=0.67)$.

Most data on the surgical treatment of rectal NECs come from a series of cases presentations. Smith (21) has reported the clinical outcomes of 126 patients with NEC, the mean OS was 27 months and 20.3 months with or without resection, no statistical significance was found.
Aytac (13) has described 25 patients with colorectal NEC from 1993 to 2011, indicating that the resection of the primary site was not able to change the poor prognosis. Notably, our analysis found that patients underwent the resection of primary site was an important factor for the prognosis prediction. Furthermore, as the detection and intervention methods of the treatment strategies have been changing very rapidly during the past two decades (22), PRRT and LAR prove to have a place for palliative purposes in patients with NEC. However, data regarding the specific effectiveness of various therapies derive from case reports and small retrospective studies $(10,23)$. Poor prognosis and proliferative nature of rectal NEC force clinical physicians to administer multidisciplinary treatment modalities (24). Currently, no standard oncological treatment strategies have been defined for rectal NEC. Thus, a systematic review of the analysis of the treatment strategies is needed in further studies.

Our study still has some limitations. First, several known prognostic factors such as Ki-67 index and WHO grade were not captured from the SEER database; the second limitation is the retrospective nature of this study. In order to confirm the prognosis predictive results, large RCT study may be still required. In addition, there are many other factors which also influence the prognosis, such as treatment modality, and further research is still warranted to improve the quality of nomogram. However, the size of the present study, which we believe to be the largest in the field of rectal NECs to date, provides a comprehensive epidemiologic picture of rectal NECs. More studies are still required to verify the characteristics.

In summary, our present study concluded that tumor differentiation, surgery and TNM stage were independent prognostic factors for survival of the rectal NEC patients. Moreover, nomograms based on both SEER data and our data were developed in this study to reveal good discrimination capability to predict different OS rates. In the future, the more advanced predictive model for this disease will be obtained to assist in risk stratification through the formulated nomogram.

\section{Supplementary data}

This is linked to the online version of the paper at https://doi.org/10.1530/ EC-18-0353.

\section{Declaration of interest}

The authors declare that there is no conflict of interest that could be perceived as prejudicing the impartiality of the research reported.

This work is licensed under a Creative Commons Attribution-NonCommercial-NoDerivatives 4.0 International License. 
Funding

This work was supported by the National Natural Science Foundation of China (81572294 and 81572298).

\section{References}

1 Dasari A, Shen C, Halperin D, Zhao B, Zhou S, Xu Y, Shih T \& Yao JC. Trends in the incidence, prevalence, and survival outcomes in patients with neuroendocrine tumors in the united states. JAMA Oncology 20173 1335-1342. (https://doi.org/10.1001/ jamaoncol.2017.0589)

2 Huguet I, Grossman AB \& O'Toole D. Changes in the epidemiology of neuroendocrine tumours. Neuroendocrinology 2017 104 105-111. (https://doi.org/10.1159/000441897)

3 Yao JC, Hassan M, Phan A, Dagohoy C, Leary C, Mares JE, Abdalla EK, Fleming JB, Vauthey JN, Rashid A, et al. One hundred years after 'carcinoid': epidemiology of and prognostic factors for neuroendocrine tumors in 35,825 cases in the United States. Journal of Clinical Oncology 200826 3063-3072. (https://doi.org/10.1200/ JCO.2007.15.4377)

4 Oberg K, Modlin IM, De Herder W, Pavel M, Klimstra D, Frilling A, Metz DC, Heaney A, Kwekkeboom D, Strosberg J, et al. Consensus on biomarkers for neuroendocrine tumour disease. Lancet Oncology 2015 16 e435-e446. (https://doi.org/10.1016/S1470-2045(15)00186-2)

5 Yachida S, Vakiani E, White CM, Zhong Y, Saunders T, Morgan R, de Wilde RF, Maitra A, Hicks J, DeMarzo AM, et al. Small cell and large cell neuroendocrine carcinomas of the pancreas are genetically similar and distinct from well-differentiated pancreatic neuroendocrine tumors. American Journal of Surgical Pathology 2012 36 173-184. (https://doi.org/10.1097/PAS.0b013e3182417d36)

6 Furumoto K, Kogire M \& Iizuka N. Long-term survival of a patient with rectal neuroendocrine carcinoma after treatment with curative resection and adjuvant chemotherapy with capecitabine. Gan to Kagaku Ryoho 201542 2485-2488.

7 Jin-Hu F, Yu-Qing Z, Su-Sheng S, Yuan-Jia C, Xing-Hua Y, Li-Ming J, Shao-Ming W, Li M, Yu-Tong H, Chang-Yan F, et al. A nation-wide retrospective epidemiological study of gastroenteropancreatic neuroendocrine neoplasms in china. Neuroendocrinology 20178 71699-71708. (https://doi.org/10.18632/oncotarget.17599)

8 Fraenkel M, Kim M, Faggiano A, de Herder WW \& Valk GD. Incidence of gastroenteropancreatic neuroendocrine tumours: a systematic review of the literature. Endocrine-Related Cancer 201421 R153-R163. (https://doi.org/10.1530/ERC-13-0125)

9 Zhou H, Zhang Y, Song Y, Tan W, Qiu Z, Li S, Chen Q \& Gao S. Marital status is an independent prognostic factor for pancreatic neuroendocrine tumors patients: an analysis of the Surveillance, Epidemiology, and End Results (SEER) database. Clinics and Research in Hepatology and Gastroenterology 201741 476-486. (https://doi. org/10.1016/j.clinre.2017.02.008)

10 Sawano M, Esaki M \& Maehata Y. Rectal neuroendocrine carcinoma associated with long-standing ulcerative colitis. Digestive Endoscopy 201628 611. (https://doi.org/10.1111/den.12640)

11 Pavel M, Baudin E, Couvelard A, Krenning E, Oberg K, Steinmuller T, Anlauf M, Wiedenmann B \& Salazar R. ENETS Consensus Guidelines for the management of patients with liver and other distant metastases from neuroendocrine neoplasms of foregut, midgut, hindgut, and unknown primary. Neuroendocrinology 201295 157-176. (https://doi.org/10.1159/000335597)

12 O'Toole D. Current trend: endocrine tumors of the stomach, small bowel, colon and rectum. Gastroenterologie Clinique et Biologique 2006 30 276-291.

13 Aytac E, Ozdemir Y \& Ozuner G. Long term outcomes of neuroendocrine carcinomas (high-grade neuroendocrine tumors) of the colon, rectum, and anal canal. Journal of Visceral Surgery 2014 151 3-7. (https://doi.org/10.1016/j.jviscsurg.2013.12.007)

14 Jilesen AP, van Eijck CH, In'T HK, van Dieren S, Gouma DJ \& Nieveen VDE. Postoperative complications, in-hospital mortality and 5-year survival after surgical resection for patients with a pancreatic neuroendocrine tumor: a systematic review. World Journal of Surgery 201640 729-748. (https://doi.org/10.1007/s00268-015-3328-6)

15 Modlin IM, Bodei L \& Kidd M. Neuroendocrine tumor biomarkers: from monoanalytes to transcripts and algorithms. Best Practice and Research: Clinical Endocrinology and Metabolism 201630 59-77. (https://doi.org/10.1016/j.beem.2016.01.002)

16 McMullen T, Al-Jahdali A, de Gara C, Ghosh S, McEwan A \& Schiller D. A population-based study of outcomes in patients with gastrointestinal neuroendocrine tumours. Canadian Journal of Surgery 201760 192-197. (https://doi.org/10.1503/cjs.007616)

$17 \mathrm{Xu}$ J, Chen Y, Chen H, Hong Z, Shi Z, Zhuo S, Zhu X \& Chen J. Identifying the neck margin status of ductal adenocarcinoma in the pancreatic head by multiphoton microscopy. Scientific Reports 20177 4586. (https://doi.org/10.1038/s41598-017-04771-w)

18 Iasonos A, Schrag D, Raj GV \& Panageas KS. How to build and interpret a nomogram for cancer prognosis. Journal of Clinical Oncology 200826 1364-1370. (https://doi.org/10.1200/ JCO.2007.12.9791)

19 Rindi G, Petrone G \& Inzani F. The 2010 WHO Classification of Digestive Neuroendocrine Neoplasms: a critical appraisal four years after Its Introduction. Endocrine Pathology 201425 186-192. (https:// doi.org/10.1007/s12022-014-9313-z)

20 Jung HJ, Hong SJ, Han JP, Kim HS, Jeong GA, Cho GS, Kim HK, Ko BM \& Lee MS. Long-term outcome of endoscopic and surgical resection for foregut neuroendocrine tumors. Journal of Digestive Diseases 201516 595-600. (https://doi.org/10.1111/17512980.12279)

21 Smith JD, Reidy DL, Goodman KA, Shia J \& Nash GM. A retrospective review of 126 high-grade neuroendocrine carcinomas of the colon and rectum. Annals of Surgical Oncology 201421 2956-2962. (https:// doi.org/10.1245/s10434-014-3725-3)

22 Wang Y, Wang W, Jin K, Fang C, Lin Y, Xue L, Feng S, Zhou Z, Shao C, Chen $\mathrm{M}$, et al. Somatostatin receptor expression indicates improved prognosis in gastroenteropancreatic neuroendocrine neoplasm, and octreotide long-acting release is effective and safe in Chinese patients with advanced gastroenteropancreatic neuroendocrine tumors. Oncology Letters 201713 1165-1174. (https://doi.org/10.3892/ol.2017.5591)

23 Zhang HP, Wu W, Yang S \& Lin J. Endoscopic treatments for rectal neuroendocrine tumors smaller than $16 \mathrm{~mm}$ : a meta-analysis. Scandinavian Journal of Gastroenterology 201651 1345-1353. (https:// doi.org/10.1080/00365521.2016.1200140)

24 Miyamae Y, Shimizu H, Naganuma A, Aiba M, Tanaka T, Ogawa T, Ogawa A \& Osamura Y. A case of rectal neuroendocrine carcinoma with metachronous liver metastasis treated with multimodality therapy. Gan to Kagaku Ryoho 201643 1009-1013.

Received in final form 14 September 2018

Accepted 20 September 2018

Accepted Preprint published online 20 September 2018 https://ec.bioscientifica.com

https://doi.org/10.1530/EC-18-0353
() 2018 The authors Published by Bioscientifica Ltd

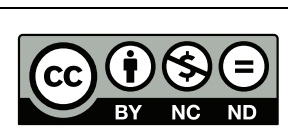

This work is licensed under a Creative Commons Attribution-NonCommercial-NoDerivatives 4.0 International License. 